\title{
The Association Between Noncontact Injuries and the Acute-Chronic Workload Ratio in Elite-Level Athletes: A Critically Appraised Topic
}

\author{
Natalie L. Myers, Guadalupe Mexicano, and Kristin V. Aguilar
}

\begin{abstract}
Clinical Scenario: Workload monitoring and management of an athlete is viewed by many as an essential training component to determine if an athlete is adapting to a training program and to minimize injury risk. Although training workload may be measured a variety of different ways, session rate of perceived exertion (sRPE) is often used in the literature due to its clinical ease. In recent years, sports scientists have been investigating SRPE as a measure of internal workload and its relationship to injury in elite-level athletes using a metric known as the acute:chronic workload ratio (ACWR). This critically appraised topic was conducted to determine if internal workload using the ACWR is associated with injury. Focused Clinical Question: In elite-level athletes, is there an association between the ACWR for sRPE and noncontact injuries? Summary of Search, Best Evidence Appraised, and Key Findings: The literature was searched for studies investigating the association between noncontact injuries and the sRPE ACWR in elite athletes. Three prospective cohort studies were included. Two studies found that high ACWR led to 2.0 to 4.5 times greater injury risk compared with a more balanced ACWR. One study found that low chronic workloads coupled with a low ACWR were associated with injury. Clinical Bottom Line: The majority of evidence suggests that when the acute workload exceeds the chronic workload, there is an increase in injury risk. The evidence also supports that a low chronic workload with a low ACWR should be considered as an injury risk factor. Strength of Recommendation: Based on the American Family Physician's Strength of Recommendation Taxonomy, there is level A evidence to support the sRPE ACWR as a risk factor for noncontract injuries in elite athletes.
\end{abstract}

Keywords: rate of perceived exertion, internal workload, training load

\section{Clinical Scenario}

Workload monitoring and management of an athlete is often implemented by coaches and health care professionals as an essential training component. It is important to address workload to determine if an athlete is adapting to a training program, as training maximizes performance while trying to minimizing injury risk. Training workload can be measured a variety of different ways, assessing both external and internal workload parameters. Internal workload is an individual's response to an external stimulus. ${ }^{1}$ A common measure of internal workload is session rate of perceived exertion (sRPE). sRPE is quantified by multiplying training session duration (in minutes) by rate of perceived exertion (RPE), a subjective index of effort often measured on a scale of 0 to 10. RPE is a valid measure and can be easily implemented into any clinical setting, making it a desirable form of measuring internal workload. ${ }^{2,3}$ In recent years, sports scientists have been investigating sRPE and its relationship to injury in elite-level athletes using a metric known as the acute: chronic workload ratio (ACWR). ${ }^{4}$ This critically appraised topic (CAT) was conducted to determine if internal workload (sRPE) using the ACWR is associated with noncontact injuries.

\section{Focused Clinical Question}

In elite-level athletes, is there an association between the ACWR for sRPE and noncontact injuries?

The authors are with the Department of Health and Human Performance, Texas State University, San Marcos, TX. Myers (natalie.myers@txstate.edu) is corresponding author.

\section{Summary of Search, Best Evidence Appraised, and Key Findings}

- The literature was searched for studies that investigated the relationship between injury risk/odds and internal workload using the ACWR

- Studies that documented noncontact injuries

- Studies that included sRPE to calculate internal workload

- Three prospective cohort studies were included

- The ACWR was defined using the following parameters in each study: a player's acute workload (most recent 1-wk training load) divided by the chronic workload (4-wk rolling average of training load)

- All 3 studies ${ }^{4-6}$ provide evidence for the ACWR (measured using sRPE) as a risk factor for noncontact injuries in elite athletes

\section{Clinical Bottom Line}

In this group of elite athletes, the majority of evidence suggests that when the acute workload exceeds the chronic workload (high ACWR), there is an increase in injury risk. The evidence also supports that low chronic load coupled with low ACWR should be considered as an injury risk factor.

\section{Strength of Recommendation}

Based on the American Family Physician's Strength of Recommendation Taxonomy, ${ }^{7}$ there is level A evidence to support sRPE ACWR as a risk factor for noncontact injuries in elite athletes. 


\section{Search Strategy}

\section{Terms Used to Guide Search Strategy}

- Patient/Client: elite athletes

- Intervention: acute:chronic workload ratio, internal workload, session rate of perceived exertion (sRPE)

- Comparison: none

- Outcome: injury risk

\section{Sources of Evidence Searched}

- SPORTDiscus

- MEDLINE

- EBSCOhost

- PubMed

- CINAHL Complete

- Additional sources found via reference lists

\section{Inclusion and Exclusion Criteria}

\section{Inclusion Criteria}

- In accordance with the Centre of Evidence-Based Medicine, level 3 evidence or higher

- Studies using noncontact injuries as the primary outcome measure

- Studies investigating sRPE workload. This measure of workload was used to calculate ACWRs using 1:4-week time period criteria

- Studies written in the English language

- Studies published in the last 5 years (2014-2018)

\section{Exclusion Criteria}

- Studies that did not use the 1:4 ACWR time frame

- Studies that included external workload variables, such as GPS metrics

- Studies that included all injuries or did not specify the type of injuries included

\section{Results of Search}

The search yielded a total of 9 possible peer-reviewed articles based on title and abstract alone. Further review identified 3 articles that met the inclusion criteria (Table 1).

\section{Best Evidence}

The studies included in this appraisal were identified as the best evidence available. Specific characteristics of each of the studies

\section{Table 1 Summary of Study Designs of Articles Retrieved}

\begin{tabular}{llcl}
\hline $\begin{array}{l}\text { Level of } \\
\text { evidence }\end{array}$ & Study design & $\begin{array}{l}\text { Number } \\
\text { located }\end{array}$ & Reference \\
\hline 3 & Prospective cohort & 3 & $\begin{array}{l}\text { Colby et } \mathrm{al}^{5} \\
\text { Hulin et } \mathrm{al}^{4} \\
\text { McCall et } \mathrm{al}^{6}\end{array}$ \\
\hline
\end{tabular}

are identified in Table 2. These studies were selected because they included sRPE ACWR using 1:4-week time frame criteria and noncontact injuries as the primary outcome.

\section{Implications for Practice, Education, and Future Research}

The goal of this CAT was to critically appraise the current evidence on the association between SRPE ACWR and noncontact injuries. Two articles in this CAT suggest that greater increases in acute workload relative to chronic workload (high ACWR) lead to greater injury likelihoods. ${ }^{4,6}$ The third study suggests low chronic load coupled with a low internal ACWR should also be considered as a risk factor for noncontact injuries, as this may lower the chronic load foundation. ${ }^{5}$ Each study was characterized as level 3 evidence according to the Centre of Evidence-Based Medicine's 2011 level of evidence table. In addition, all 3 studies were found to have a grade A strength of recommendation, according to the American Family Physician's Strength of Recommendation Taxonomy. The results from this CAT suggest that monitoring sRPE, and using chronic load and the ACWR metric for the interpretation of workload, should be incorporated into training regimes to reduce noncontact injury risk. Self-perceived RPE is a measure that can be easily collected by health care professionals interested in monitoring internal workload, ${ }^{2}$ and the ACWR can be used to help determine an athlete's preparedness for activity.

All 3 studies support that chronic load is an important factor in the workload-injury relationship. Low chronic workloads coupled with low ACWR are associated with injury. In addition, the findings of this CAT suggest high ACWR leads to 2.0 to 4.5 times greater injury risk compared with a more balance ACWR. Researchers investigating the workload-injury relationship have suggested that injury likelihood is low when the ACWR is between 0.8 and 1.3. ${ }^{8}$ The evidence presented in this CAT supports this relationship. However, it should be noted that a ratio between 0.8 and 1.3 does not necessarily mean the likelihood of injury does not exist. There may be athletes within this range who still sustain an injury; thus, other factors associated with injury should be considered, such as previous injury history, strength, psychosoical factors, and aerobic capacity.

These studies are not without limitations. First, none of the studies calculated statistical power prior to analyzing the workload-injury relationships, potentially introducing threats to the internal validity of the studies. Second, 2 of these included football players as the sample population, while the third included cricket bowlers. Until more data are available on a variety of sports, applying ACWR recommendations should be done with caution. Future research should investigate this metric in both team sports and individual-based sports. Finally, sRPE measures were only calculated for practice and game training; further research may be needed to investigate workload with respect to resistance and crosstraining activities.

In conclusion, there is an association between noncontact injuries and sRPE ACWR (1:4 wk) in elite-level footballers and cricket bowlers. sRPE acute and chronic workloads should be monitored and managed to diminish the likelihood of noncontact injuries. sRPE is an easy-to-use validated scale that requires minimal effort on the part of the athlete. Overall, athletes should consider building high chronic workloads in an appropriate sequence to reduce the likelihood of noncontact injuries. 
Table 2 Characteristics of Included Studies

\begin{tabular}{llll}
\hline Authors & Colby et $\mathbf{a l}^{\mathbf{5}}$ & Hulin et al $^{\mathbf{4}}$ & McCall et al $^{\mathbf{6}}$ \\
\hline Study design & Prospective cohort & Prospective cohort & Prospective cohort \\
Participants & 70 Australian footballers & 28 cricket fast bowlers (26 [5] y) & 171 football players \\
& $(23[3]$ y) from 1 Australian & contracted to one of 2 teams: New & $(25[5]$ y) from \\
& Football League across & South Wales or Victorian cricket & 5 European teams during \\
& 4 consecutive seasons & squad between 2006 and 2012. Of & 2015/2016 competitive season
\end{tabular}
the 5 seasons, $11 \%$ (3) played 3 seasons, 33\% (9) played 2 seasons, and $57 \%$ (16) played 1 season

Intervention investigated

Outcome measure

Main findings

Level of evidence

3

Validity score: modified Downs and Black

Conclusion
Any lower body noncontact injury resulting in matches missed

Internal workload: quantified using a subjective 10-point modified Borg scale sRPE.

Estimate of internal workload: RPE multiplied by session duration (in min) or training or match session

ACWR: A player's acute (1 wk) workload divided by their chronic (4-wk rolling average) workload

97 noncontact lower body injuries were sustained across the 4 seasons (9.8 injuries per $1000 \mathrm{~h}$ ) A low sRPE chronic load coupled with a low sRPE ACWR was associated with increased injury risk in the subsequent week (adjusted incidence risk ratio $=$ 2.52; 95\% CI, 1.01-6.29)

Low chronic workloads coupled with a low ACWR area associated with noncontact injuries, compared with high chronic loads and a moderate ACWR in elite Australian footballers
Any noncontact injury that resulted in a loss of either match time or greater than one training session over a 1 -wk period

Acute workload higher than the chronic workload was associated with injury risk in the subsequent week $(\mathrm{RR}=2.2 ; 95 \% \mathrm{CI}$, 1.91-2.53)

Fast bowlers with an internal ACWR of greater than $200 \%$ were at 4.5 times greater RR of injury compared with a more balanced ACWR between 50\% and $99 \%$

3

$13 / 27$

Large spikes in acute workload relative to chronic workload are associated with increased risk of noncontact injuries in elite cricket fast bowlers
Internal workload: quantified using a subjective 10-point Borg scale as an estimate of training intensity

Estimate of internal workload: RPE multiplied by session duration (in min) or training or match session

ACWR: A player's acute (1 wk) workload divided by their chronic (4-wk rolling average) workload The RPE was collected $30 \mathrm{~min}$ after completion of the session/ match

Any noncontact injury when a player was unable to fully participate in future football training or match due to physical complaints

123 noncontact time-loss injuries were recorded

Football players with an internal ACWR of $>1.38$ were at 2.13 (95\% CI, 1.20-3.77) times greater RR of injury compared with those with an ACWR between 0.60 and 0.97

3

ACWR (1:4) showed significant associations with noncontact injuries in the subsequent weeks in elite football players

Abbreviations: ACWR, acute:chronic workload ratio; CI, confidence interval; RR, relative risk; sRPE, session rate of perceived exertion.

\section{References}

1. Soligard T, Schwellnus M, Alonso JM, et al. How much is too much? (Part 1) International Olympic Committee consensus statement on load in sport and risk of injury. Br J Sports Med. 2016; 50(17):1030-1041. PubMed ID: 27535989 doi:10.1136/bjsports2016-096581

2. Foster C, Florhaug JA, Franklin J, et al. A new approach to monitoring exercise training. J Strength Cond Res. 2001;15(1):109-115. PubMed ID: 11708692
3. Foster C, Rodriguez-Marroyo JA, de Koning JJ. Monitoring training loads: the past, the present, and the future. Int J Sports Physiol Perform. 2017;12(suppl 2):S22-S28. PubMed ID: 28253038 doi:10. 1123/IJSPP.2016-0388

4. Hulin BT, Gabbett TJ, Blanch P, Chapman P, Bailey D, Orchard JW. Spikes in acute workload are associated with increased injury risk in elite cricket fast bowlers. Br J Sports Med. 2014;48(8):708-712. PubMed ID: 23962877 doi:10.1136/bjsports-2013-092524

5. Colby MJ, Dawson B, Peeling P, et al. Multivariate modelling of subjective and objective monitoring data improve the detection of 
non-contact injury risk in elite Australian footballers. J Sci Med Sport . 2017;20(12):1068-1074. PubMed ID: 28595869 doi:10.1016/j. jsams.2017.05.010

6. McCall A, Dupont G, Ekstrand J. Internal workload and non-contact injury: a one-season study of five teams from the UEFA Elite Club Injury Study. Br J Sports Med. 2018;52(23):1517-1522. PubMed ID: 29626055 doi:10.1136/bjsports-2017-098473
7. Ebell MH, Siwek J, Weiss BD, et al. Strength of recommendation taxonomy (SORT): a patient-centered approach to grading evidence in the medical literature. J Am Board Fam Pract. 2004;17(1):59-67. PubMed ID: 15014055 doi:10.3122/jabfm.17.1.59

8. Gabbett TJ. The training-injury prevention paradox: should athletes be training smarter and harder? Br J Sports Med. 2016;50(5):273280. PubMed ID: 26758673 doi:10.1136/bjsports-2015-095788 